\title{
Sédentarisation des éleveurs Mbororo et évolution de leurs pratiques au Nord Cameroun
}

\author{
Liba'a Natali Kossoumna $^{1}$ \\ Patrick Dugué ${ }^{2}$ \\ Emmanuel Torquebiau ${ }^{3}$ \\ ${ }^{1}$ Université de Ngaoundéré \\ BP 716 \\ Garoua \\ Cameroon \\ <kolibaa@yahoo.fr> \\ ${ }^{2}$ Cirad \\ UMR Innovation \\ TA-C 85/15 \\ 73, rue J-F Breton \\ 34398 Montpellier cedex 5 \\ France \\ <patrick.dugue@cirad.fr> \\ ${ }^{3}$ Cirad \\ UR AGIRS \\ P.O. BOX 26884, Monument Park \\ 0105 Pretoria \\ Afrique du Sud \\ <emmanuel.torquebiau@cirad.fr>
}

\begin{abstract}
Résumé
Au nord du Cameroun, la croissance de la population rurale accroit la concurrence entre éleveurs et agriculteurs pour l'accès aux ressources naturelles. Les décideurs souhaitent voir les éleveurs se sédentariser avec leurs troupeaux et abandonner leur pratique de transhumance. En fait, si on constate bien une fixation de l'habitat des éleveurs, ceux-ci maintiennent la transhumance saisonnière des troupeaux. Les principales raisons de cette semi-sédentarisation des éleveurs Mbororo et de leur famille sont d'ordre économique mais surtout social : reconnaissance des campements d'éleveurs comme village, revendication d'infrastructures (forage, école), acquisition de droits fonciers. La poursuite de la pratique de la transhumance pour une partie du cheptel permet une exploitation raisonnée dans le temps et l'espace des ressources fourragères et résout le problème de l'exigüité des espaces disponibles pour l'élevage. Du point de vue économique, cette forme de sédentarisation rapproche ces éleveurs des marchés du bétail et des sources d'information. Ces systèmes de production semblent performants dans la mesure où les unités de production sont autosuffisantes en céréales et en lait et dégagent un solde monétaire. Leur durabilité dépend du maintien de la transhumance et donc de la disponibilité de grands parcours situés hors des territoires de sédentarisation actuels.
\end{abstract}

Mots clés : agriculteur ; Cameroun ; développement durable ; éleveur ; savane ; sédentarisation ; transhumance.

Thèmes : productions animales ; ressources naturelles et environnement.

\section{Abstract \\ Change of practices by settling transhumant Mbororo farmers in Northern Cameroon}

In Northern Cameroon, human population growth leads to increased competition for the same natural resources between nomadic livestock breeders (pastoralists) and crop farmers. While decision-makers would prefer pastoralists to settle and give up nomadism and transhumance, what is observed is a partial settling process with permanent dwellings and crop fields but with the persistence of seasonal practices of transhumance for a large part of the animals. The main reason behind this semi-settlement process is of both a social and economic nature: Pastoralists villages are given an official status by the authorities, thus engendering requests for public infrastructures such as schools and boreholes as well as claims for tenure rights. Seasonal transhumance for part of the herds renders possible sustainable temporal and spatial use of fodder resources and solves land availability problems. From the economic point of view, this situation opens market opportunities for pastoralists. Cereal and milk yields are good and the access to local markets contributes to the good performance of those semi-settled production systems. Transhumance is a key factor in their sustainability and is conditioned by the availability of grazing lands beyond settling areas.

Key words: Cameroon; farmers; livestock breeders; sustainable development; savannas; sedentarization; transhumance.

Subjects: animal resources; natural resources and environment.

Tirés à part : LN Kossoumna 
$\mathrm{E}$ n Afrique soudano-sahélienne, l'élevage extensif transhumant est souvent considéré par les décideurs politiques et les techniciens agricoles comme une activité dégradant l'environnement. Avec la croissance continue de la population rurale et l'augmentation des besoins en terres, ces décideurs souhaitent donc que les éleveurs se sédentarisent afin de limiter leur emprise spatiale et renoncent à la transhumance. Toutefois, les pasteurs ont maintenu cette pratique d'élevage extensive tout en faisant évoluer leurs systèmes de production. Ainsi ils développent la production céréalière intensive autour de l'habitat fixé tout en continuant à faire transhumer, à certaines saisons, une partie du cheptel (Dugué et al., 2004). L'argumentaire des politiques publiques n'est donc pas en phase avec les réalités du terrain (Boutrais, 1996 ; Pourtier, 2003). Afin d'explorer et de comprendre les tenants et aboutissants de ces écarts, notre objectif ici est d'analyser les changements en cours au sein des communautés d'éleveurs et de leurs territoires d'activités dans le bassin de la Bénoué, dans la province du Nord au Cameroun. Dans un premier temps, les changements survenus dans les modes de vie et de production des éleveurs seront caractérisés, notamment ceux liés à la fixation de l'habitat, à la diversification des activités et à la mobilité du troupeau. Ensuite, l'intérêt de préserver la mobilité du bétail dans une perspective de développement durable de cette région sera discuté.

\section{Quels espaces pour les éleveurs?}

Au Nord Cameroun l'élevage bovin des Peuls (Mbororo et Fulbé) est conduit de façon extensive sur les parcours naturels, sans recourir à l'achat massif d'aliments du bétail ni à des pratiques volontaristes de gestion des parcours (mise en défens ou ensemencement des espaces dégradés, contrôle des feux, etc.). Cet élevage bovin est en expansion continue depuis une trentaine d'années dans les provinces du Nord et de l'Extrême-Nord (figure 1). Divers auteurs ont montré que durant cette période le ratio bovin/surface en parcours avait été multiplié par huit pour l'ensemble de ces deux provinces, du fait d'une augmentation du cheptel bovin, mais aussi de la surface cultivée au détriment des parcours (Donfack et al., 1997) (Labonne et al., 2003). Cette dernière évolution est plus marquée dans la province du Nord où le taux de croissance démographique rurale dépassait, pour cette période, $6 \%$ par an, l'immigration constituant plus de la moitié du croît.

Pour corriger l'inégale répartition de la population entre les deux provinces septentrionales du pays, l'État camerounais a mis en place entre 1966 et 1986 plusieurs programmes d'appui à la migration de familles paysannes des zones les plus peuplées de l'Extrême-Nord vers le bassin de la Bénoué dans la Province du Nord pour mettre en valeur des terres non encore défrichées. Ces programmes ont surtout favorisé l'agriculture et plus particulièrement la production cotonnière. Même si quelques pistes à bétail ont été délimitées dans cette région, l'élevage a été peu pris en compte dans les programmes d'aménagement et de colonisation des zones d'accueil. Les attributions foncières aux agriculteurs migrants ont été gérées par les autorités coutumières villageoises et les seuils d'acceptabilité des populations humaine et animale n'ont jamais été définis par les projets ou l'administration (Seignobos et al., 2002). Le bassin de la Bénoué a donc été rapidement mis en culture, avec comme conséquence pour les éleveurs la réduction des superficies des parcours (burum) et de la largeur des pistes à bétail (burtol).

De plus, les aires protégées -3 parcs nationaux, 28 zones d'intérêt cynégétique (ZIC) - occupent près de $45 \%$ de la superficie de la Province du Nord (figure 1). Ces aires protégées, vaste ensemble de savane arborée, sont interdites au pâturage et à l'agriculture. Leur maintien en l'état fait l'objet de tensions

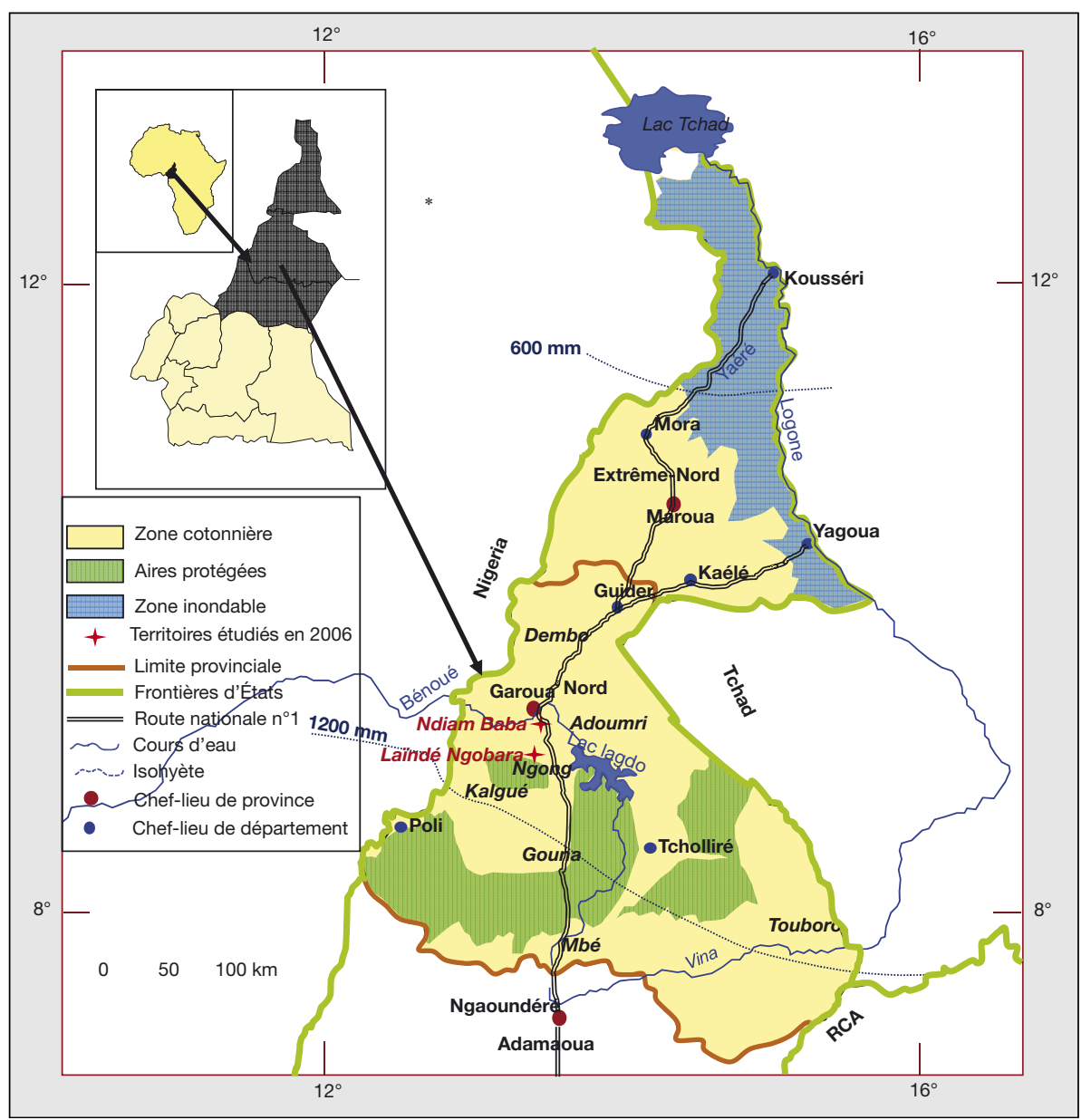

Figure 1. Provinces du Nord et de l'Extrême-Nord du Cameroun.

Figure 1. North and Far North of Cameroon. 
entre le "lobby " agricole, celui de l'élevage et les services de l'environnement. Les éleveurs sont donc "bloqués " entre ces zones protégées et les $15 \%$ de la Province occupés par les cultures. Ils n'ont pas d'autre option que de faire pâturer leurs troupeaux en saison des pluies dans les rares grands parcours (burum) ou dans les zones marginales de collines, mais aussi dans certaines zones d'intérêt cynégétique mal gardées. En saison sèche, ils privilégient la vaine pâture des résidus de culture. En 2002, Labonne et al. (2002) considéraient que les superficies disponibles respectivement pour l'élevage et l'agriculture semblaient suffisantes mais que leur accessibilité et leur répartition dans l'espace posaient problème.

\section{Méthodologie}

Notre zone d'étude comprend la région du bassin de la Bénoué et deux villages d'éleveurs Mbororo : Ndiam Baba créé en 1981 et Laïndé Ngobara créé en 1995. La communauté Mbororo est une des composantes du grand groupe ethnique Peul d'Afrique subsaharienne. Ces deux villages constituent le territoire d'attache où se sont sédentarisés ces éleveurs. Ils y disposent d'une superficie respective de 220 et 174 hectares qu'ils gèrent euxmêmes et qui leur a été affectée par l'autorité coutumière (le lamido de Tchéboa dans les deux cas) avec l'accord de l'Administration. Ce choix permet de comparer deux dates de sédentarisation et deux situations, l'une proche d'une zone urbaine (Ndiam Baba situé à 12 kilomètres de Garoua) et l'autre au cour d'une zone très agricole de production cotonnière (Laïndé Ngobara situé à environ 40 kilomètres au sud de Garoua).

Quatre niveaux d'analyse ont été pris en compte dans cette étude menée en 2006 et 2007 (Kossoumna Liba'a, 2008) :

- la région du bassin de la Bénoué où s'organise la transhumance des troupeaux afin de comprendre les évolutions de cette pratique ;

- la petite région comprenant les deux villages Mbororo et les territoires d'agriculteurs et d'éleveurs adjacents. Nous y avons analysé les complémentarités et les compétitions entre les différents acteurs ;

- le territoire d'attache des éleveurs pour lesquels on a caractérisé les systèmes de mise en valeur des ressources naturelles par l'élevage et l'agriculture ;

- l'unité de production (UP), afin de mettre en évidence les évolutions des systèmes de production et des pratiques des éleveurs. Pour cela, 27 UP ont été suivies pendant une année complète et réparties selon quatre types: i) jeunes éleveurs gérant peu de bovins et de petites surfaces cultivées (type 1) ; ii) éleveurs gérant un troupeau et des surfaces cultivées importantes (type 2); iii) éleveurs âgés gérant peu de bovins et peu de surface cultivée (type 3) ; iv) éleveurs gérant un grand troupeau, sans terres cultivées (type 4).

Le déroulement de ces travaux a été perturbé durant l'année 2007 par la recrudescence d'exactions de bandes armées contre les familles d'éleveurs Mbororo : kidnapping d'enfants et demande de rançons, vol de bétail. Des entretiens avec les familles concernées par ces phénomènes de violence ont permis de caractériser leurs impacts sur les pratiques et systèmes d'élevage.

\section{Résultats}

\section{Maintien de la transhumance}

Si le nomadisme a disparu dans le bassin de la Bénoué, la transhumance des troupeaux des éleveurs Mbororo y est encore très vivace. L'ensemble du cheptel géré par ces éleveurs ne réside dans les territoires d'attache qu'à deux périodes précises de l'année : pour la vaine pâture des résidus de culture (décembre-janvier) de leurs champs et des parcelles des agriculteurs des territoires voisins, et durant la courte période de retour de Mayo Bocki (fin juin-début juillet) qui permet aux éleveurs de terminer de fumer leurs champs avant le labour. Généralement, chaque UP conserve dans le territoire d'attache durant toute l'année un noyau d'élevage (moins de 15 bovins) pour la production de lait et les travaux culturaux. Divers lieux et types de transhumance sont distingués :

- la petite transhumance concerne d'abord les petits troupeaux des UP des types 1 et 3 . Elle se pratique à moins de 20 kilomètres du territoire d'attache dans des zones de collines incultes proches de Ndiam Baba (juillet-septembre) et le long des berges de la Bénoué (mars-mai);
- la grande transhumance intéresse de plus grands troupeaux (types 2 et 4) et valorise des parcours plus vastes partagés avec un nombre important d'éleveurs : burum de Mayo Bocki au sud, dès les premières pluies d'avril à la mijuin ; burum de Kalgué à l'ouest, délimité par le service de l'élevage, et la région de Dembo au nord, peu peuplée, du fait du relief, durant la période mi-juillet à fin octobre.

Le maintien de la transhumance demeure la stratégie de base pour s'adapter à la forte inégalité spatio-temporelle des ressources alimentaires pastorales. Il s'explique avant tout par l'insuffisance de la production fourragère dans les territoires d'attache pour nourrir le bétail (1 022 bovins à Ndiam Baba et 1756 à Laïndé Ngobara). Si on prend comme base de calcul qu'un parcours naturel peut produire au mieux 2,5 tonnes/hectare/an de biomasse fourragère (matière sèche) consommable par les bovins, qu'un hectare de maïs bien fertilisé fournit 5 tonnes/hectare de paille consommable, et qu'un bovin adulte consomme 6,5 kg de biomasse (matière sèche) par jour on peut estimer que ces territoires peuvent alimenter respectivement environ 300 bovins à Ndiam Baba et 230 à Laïndé Ngobara (tableau 1). Vu les effectifs recensés, le recours à la transhumance est indispensable à l'entretien du bétail. Le développement de la culture fourragère dans ces territoires d'attache ne pourrait pas apporter un surplus d'alimentation suffisant à moins de recourir à des systèmes de culture très intensifs, non compatibles avec les moyens des éleveurs (irrigation et fertilisation, etc.).

\section{Fixation de l'habitat et développement des relations intercommunautaires}

Les éleveurs étudiés ont quitté le Nigeria en 1969 pour s'installer dans le lamidat de Tchéboa. À cette époque, ils ne faisaient que traverser cette région où ils résident actuellement pour transhumer au bord de la Bénoué (figure 1). C'est en 1981 que le premier Mbororo s'est fixé sur le site actuel de Ndiam Baba. Les éleveurs de Laïndé Ngobara se sont sédentarisés dans ce site en 1995 après avoir essayé de s'installer dans des localités plus proches de Garoua au début des années 1990. Les principales raisons de la fixation de l'habitat et d'une partie des activités des 
éleveurs sont d'ordre social et économique. La sédentarisation amène les pouvoirs publics à reconnaitre officiellement les campements des éleveurs constitués d'habitations "en dur" en leur attribuant le statut officiel de village. Ils ont reçu des cartes d'électeurs leur permettant de voter et de se faire représenter dans les conseils locaux (communes rurales, etc.). Ainsi, ils peuvent plus facilement revendiquer des infrastructures (école, dispensaire, forage) et des appuis auprès des services publics et des projets. Mais, surtout, les éleveurs acquièrent des droits fonciers sur le territoire délimité qui leur a été affecté. C'est également une nouvelle forme de vie et de bien-être qui est recherchée par les familles d'éleveurs grâce à la présence à proximité de leur village d'une main-d'œuvre peu onéreuse d'agriculteurs pauvres en quête de travail rémunéré. Les travaux pénibles comme la construction des maisons, l'entretien des champs et parfois même la garde du troupeau sont ainsi souvent confiés à cette maind'œuvre. Du point de vue économique, la sédentarisation rapproche les éleveurs des lieux de vente des produits animaux : les marchés du bétail (Ngong, Adoumri). En fait, ces éleveurs proches des marchés tirent une partie de leurs revenus de l'achat et la revente du bétail sur des pas de temps parfois très courts et la proximité des marchés leur permet d'acquérir rapidement les informations utiles à cette pratique du commerce du bétail.

La fixation des éleveurs dans les territoires d'attache a également favorisé l'établissement de relations sociales avec les autres communautés d'éleveurs, les agriculteurs et les citadins. Ainsi, près de la moitié du bétail géré par les Mbororo des deux villages appartient à des commerçants ou fonctionnaires aisés de la ville qui leur confient des bovins. Les alliances avec les citadins facilitent l'accès aux services et en particulier l'approvisionnement en tourteau de coton sur lequel existe une forte spéculation. Enfin, la fixation de l'habitat et l'attribution de terres ont incité ces familles à développer la culture des céréales, base de leur alimentation dont le surplus éventuel est facilement commercialisable.

\section{Production céréalière et performances économiques}

Les Peuls, caractérisés par leurs savoirfaire pastoraux ont aussi donné l'exemple

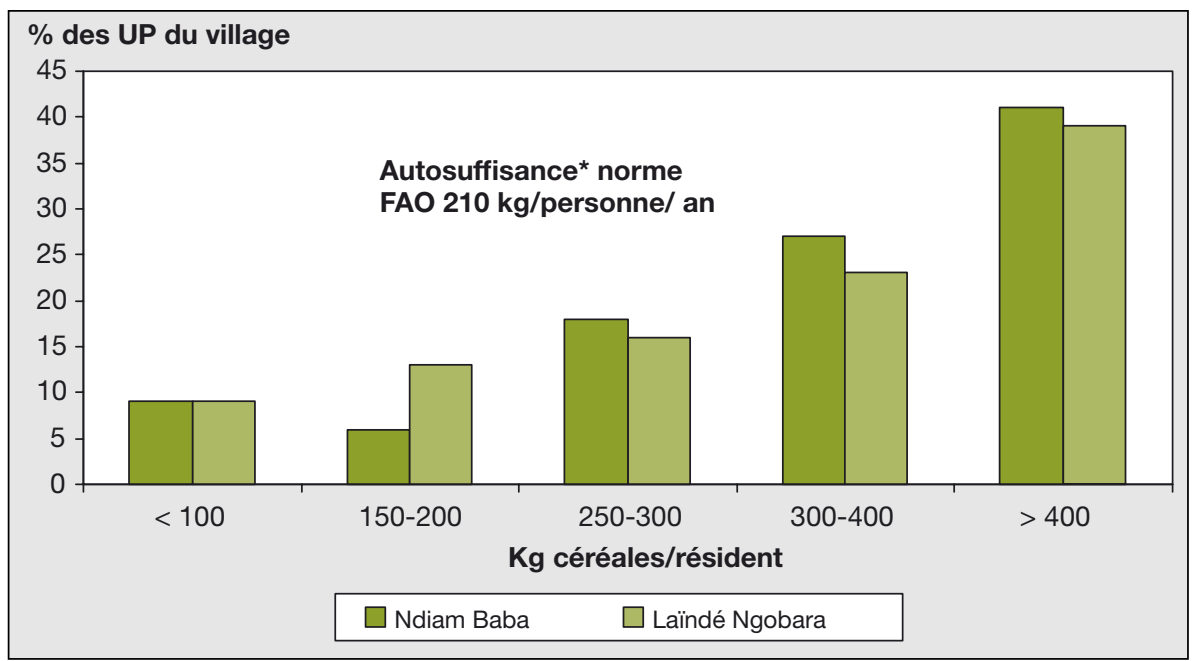

Figure 2. Production céréalière dans les deux territoires en kg/résident par an.

Figure 2. Cereal production in kg/person per year.

UP $=$ unité de production.

${ }^{(*)}$ Avec le complément alimentaire apporté par le lait, cette norme en céréale est certainement surévaluée.

de l'intégration "élevage-agriculture ", lorsque cette association était bien adaptée au contexte agraire (D’Aquino, 2000 ; Bonfiglioli, 1988; Benoit, 1988). Dans notre cas, les éleveurs Peuls Mbororo ont plus développé l'agriculture à Ndiam Baba $(0,4$ hectare cultivé par an et par résident) où l'implantation des éleveurs est plus ancienne qu'à Laïndé Ngobara $(0,15 \mathrm{ha} / \mathrm{an} / \mathrm{résident})$. La culture du maïs occupe plus de $90 \%$ des surfaces cultivées. Peu coutumiers de l'usage des engrais minéraux car non intégrés aux circuits d'approvisionnement en intrants liés à la culture cotonnière, les éleveurs valorisent bien les périodes de présence de leurs animaux dans le territoire d'attache pour assurer la fertilisation des cultures vivrières et entretenir la fertilité de leurs terres. La généralisation du parcage tournant dans les champs, facilitée par l'acquisition de fils de fer barbelé, permet d'optimiser la fumure animale produite la nuit en saison sèche. Cette pratique a modifié le système de transhumance. Autrefois, certains animaux pouvaient passer des années sans revenir dans le territoire d'attache. Ils y passent tous désormais systématiquement de un à trois mois par an pour fumer les parcelles. Ces pratiques constituent la base du maintien de la fertilité du sol chez ces éleveurs et expliquent les rendements du maïs (de 2,5 à $5 \mathrm{t} / \mathrm{ha}$ ), bien supérieurs à ceux obtenus par les agriculteurs des villages voisins (de 2 à $2,5 \mathrm{t} / \mathrm{ha}$ ).
De ce fait, plus de $80 \%$ des UP étudiées sont autosuffisantes en céréales et la plupart d'entre elles vendent des excédents (figure 2). En plus, l'analyse des flux monétaires des UP montre qu'elles dégagent un solde monétaire positif ( $\mathrm{Sm}$ ) pour l'année observée (figure 3):

$$
\begin{aligned}
{[\mathrm{Sm}=} & \sum \text { Ventes }+\sum \text { Bénéfices commerciaux } \\
& -\sum \text { Charges de production } \\
& \left.-\sum \text { Dépenses familiales }\right] .
\end{aligned}
$$

Les UP de Ndiam Baba apparaissent économiquement plus performantes que celles de Laïndé Ngobara (Sm moyens respectifs de 774000 et 329000 $\mathrm{F}$ CFA $/ \mathrm{an} / \mathrm{UP}^{1}$ ). Les recettes proviennent essentiellement de la vente des animaux (bovins, ovins, caprins) et accessoirement de la vente de céréales (Koussoumna Liba'a, 2008). Cette différence peut s'expliquer par le temps consacré par les éleveurs à Ndiam Baba aux activités d'achat et de revente de bétail, et aussi par l'ancienneté de leur sédentarisation qui leur a permis de constituer des réseaux socioprofessionnels utiles à ce commerce. Quant aux revenus issus du lait, ils apparaissent pour le moment marginaux car les éleveurs n'ont pas encore de stratégies de production et de commercialisation du lait malgré la proximité de la ville de Garoua. Le surplus de lait après l'autoconsommation familiale est laissé à la disposition des femmes qui le

\footnotetext{
$\overline{11 \text { euro }}=655,96$ F CFA.
} 


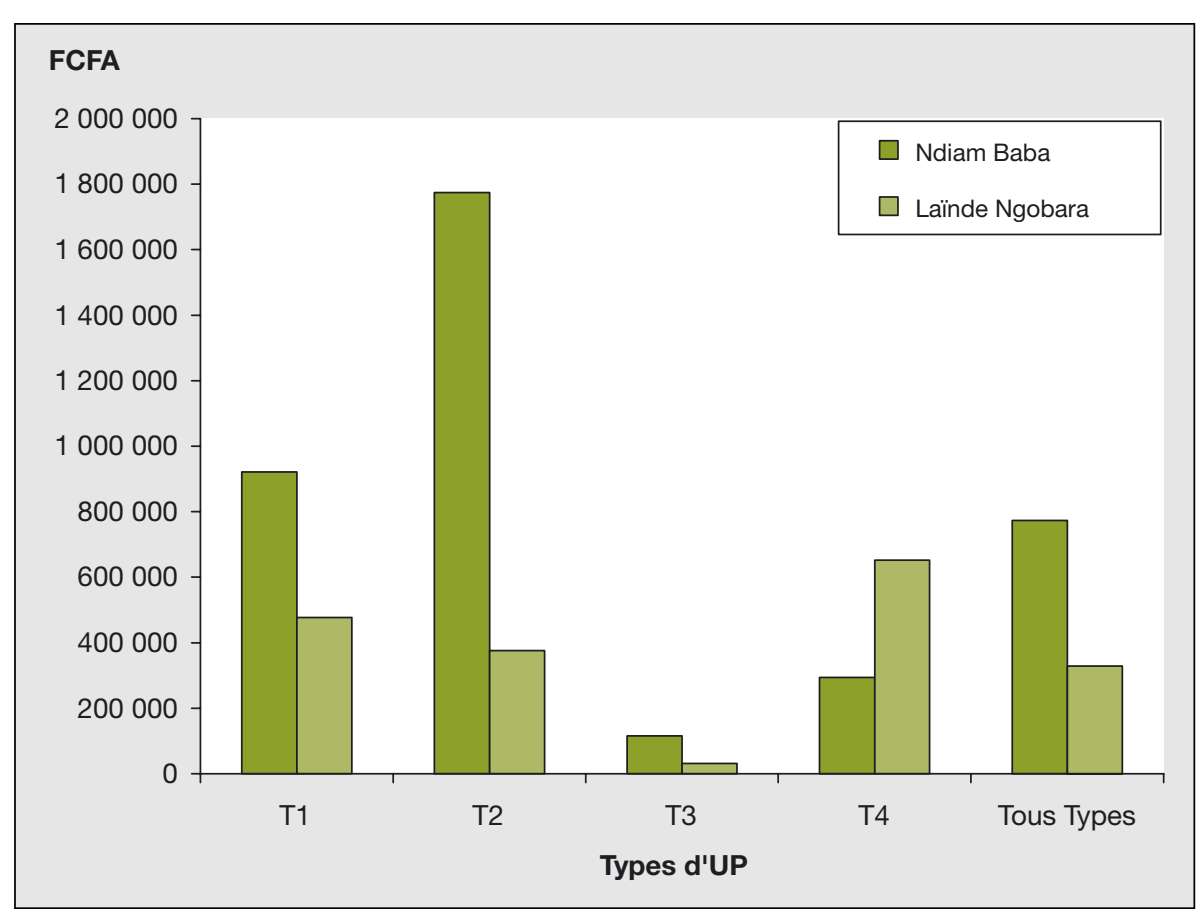

Figure 3. Solde monétaire moyen par unité de production (UP) par type et par village (en F CFA).

Figure 3. Average cash balance by UP type and territory (in F CFA).

1 euro $=655,96$ F CFA ; UP $=$ unité de production.

commercialisent individuellement, ce qui a pu amener à sous-estimer cette source de revenu dans les comptes des UP.

\section{Maintien de la transhumance}

Si le nomadisme a disparu dans le bassin de la Bénoué, la transhumance des troupeaux des éleveurs Mbororo y est encore très vivace. Divers lieux et types de transhumance sont distingués :

- la petite transhumance concerne d'abord les petits troupeaux des UP des types 1 et 3 . Elle se pratique à moins de 20 kilomètres du territoire d'attache dans des zones de collines incultes proches de Ndiam Baba (juillet-septembre) ou le long des berges de la Bénoué (mars mai) ;

- la grande transhumance intéresse de plus grands troupeaux (types 2 et 4 ) et valorise des parcours plus vastes partagés avec un nombre important d'éleveurs : burum de Mayo Bocki au sud, dès les premières pluies d'avril à la mijuin ; burum de Kalgué à l'ouest délimité par le Service de l'élevage et la région de Dembo au nord peu peuplée du fait du relief, durant la période mi-juillet à fin octobre.
L'ensemble du cheptel géré par ces éleveurs ne réside dans les territoires d'attache qu'à deux périodes précises de l'année : pour la vaine pâture des résidus de culture qui s'étend aux parcelles récoltées des territoires d'agriculteurs voisins (décembre-janvier) et durant la courte période de retour de Mayo Bocki (fin juin-début juillet) qui permet aux éleveurs de terminer de fumer leurs champs avant le labour. Généralement, chaque UP conserve dans le territoire d'attache durant toute l'année un noyau d'élevage (moins de 15 bovins) pour la production de lait et les travaux culturaux.

Le maintien de la transhumance demeure la stratégie de base pour s'adapter à la forte inégalité spatio-temporelle des ressources alimentaires pastorales. Il s'explique avant tout par l'insuffisance de la production fourragère dans les territoires d'attache pour nourrir le bétail (1 022 bovins à Ndiam Baba et 1756 à Laïndé Ngobara). Si on prend comme base de calcul qu'un parcours naturel peut produire au mieux 2,5 t/ha/an de biomasse fourragère (matière sèche) consommable par les bovins, qu'un hectare de mais bien fertilisé fournit $5 \mathrm{t} /$ ha de paille consommable, et qu'un bovin adulte consomme
6,5 kg de biomasse (matière sèche) par jour on peut estimer que ces territoires peuvent alimenter respectivement environ 300 bovins à Ndiam Baba et 230 à Laïndé Ngobara (tableau 1). Vu les effectifs recensés, le recours à la transhumance est indispensable à l'entretien du bétail. Le développement de la culture fourragère dans ces territoires d'attache ne pourrait pas apporter un surplus d'alimentation suffisant à moins de recourir à des systèmes de culture très intensifs, non compatibles avec les moyens des éleveurs (irrigation et fertilisation, etc.).

\section{Insécurité et impacts sur les pratiques}

Le grand banditisme avait été longtemps cantonné à certains grands axes routiers du nord du Cameroun où les " coupeurs de route "armés rançonnaient les voyageurs. Aujourd'hui, du fait de la valeur du bétail, les éleveurs sont également devenus une cible privilégiée de ce banditisme " rural ". Dans les cas, avérés, de kidnapping de leurs enfants, les éleveurs sont en mesure de payer des rançons de plusieurs millions de F CFA - ce que ne peuvent faire des agriculteurs sédentaires disposant d'un faible capital. Dans ce contexte, ils ont dû changer de mode de conduite de leur troupeau. Si auparavant ils exhibaient fièrement leur bétail, ils sont aujourd'hui obligés de scinder les grands troupeaux en sous-unités de 20 à 30 têtes réparties dans différentes zones de pâturage. Cet "allotement forcé " entraîne un accroissement des charges d'élevage : recours à plusieurs bergers, nombreux déplacements des chefs d'UP pour suivre l'état des troupeaux.

Suite à plusieurs prises d'otages d'enfants à Laïndé Ngobara et à proximité de Ndiam Baba durant l'année 2007, la plupart des familles d'éleveurs se sont réfugiées dans les gros bourgs agricoles situés le long de la route goudronnée. Une partie des troupeaux a rejoint le Nigeria ou d'autres contrées plus sûres, mais les éleveurs continuent à cultiver leurs champs dans les territoires d'attache. On constate ainsi un début de régression des complémentarités agriculture-élevage, avec des incidences attendues pour les éleveurs sur la fertilité de leur terre, le renchérissement de la fertilisation de leur culture de maïs et une baisse des rendements en céréales. Dans le cas d'un retour de la sécurité, de nombreux éleveurs affirment qu'ils retourneront dans leur territoire d'attache, qu'ils considèrent comme leur bien. 
Tableau 1. Occupation du sol et charge animale dans les territoires d'attache.

Table 1. Land use and animal load in livestock breeders' land.

\begin{tabular}{|c|c|c|}
\hline & Ndiam Baba & Laïndé Ngobara \\
\hline Surface totale & 220 hectares & 174 hectares \\
\hline dont - habitations & $4 \%$ & $3 \%$ \\
\hline - champs cultivés des Mbororo & $27 \%-(60$ hectares $)$ & $21 \%-(37$ hectares) \\
\hline - enclaves cultivées par les agriculteurs & $6 \%$ & $7 \%$ \\
\hline - parcours, collines... & $63 \%$ & $69 \%$ \\
\hline Population totale résidente & 150 & 249 \\
\hline Surface cultivée/résident (en hectares) & 0,40 & 0,15 \\
\hline $\begin{array}{l}\text { - Effectif bovins adultes total et présent au village [de novembre à février] } \\
\text { et [de juin à début juillet] }\end{array}$ & 1022 & 1756 \\
\hline $\begin{array}{l}\text { - Effectif bovins adultes restant au village [de mars à juin] et [de fin juillet } \\
\text { à novembre] }\end{array}$ & 220 & 317 \\
\hline - Biomasse disponible sur une année en tonnes de matière sèche & 711 & 545 \\
\hline $\begin{array}{l}\text { - Nombre de bovins adultes pouvant être nourris de façon permanente } \\
\text { dans le territoire d'attache }\end{array}$ & 300 & 230 \\
\hline
\end{tabular}

\section{Discussion}

La durabilité des systèmes de production des éleveurs Mbororo sédentarisés peut être discutée en considérant les quatre composantes définies par Landais (1998): développement économique, protection de l'environnement, développement social et humain, et lien intergénérationnel (tableau 2).

\section{Des systèmes économiquement performants}

La grande majorité des UP suivies représentant la diversité des systèmes de production d'éleveurs sédentarisés de cette région sont autosuffisantes en céréales et dégagent un revenu monétaire après paiement des principaux coûts de pro- duction et dépenses du ménage. La reconduction de ce suivi technico-économique pendant quelques années permettrait de confirmer la bonne santé économique de ces UP et leur capacité à s'adapter à des aléas climatiques et de santé animale. Du fait que la majorité des revenus monétaires proviennent de la vente des animaux adultes, la durabilité économique de ces systèmes de production est fortement liée à la taille et au niveau de productivité des

\section{Tableau 2. Durabilité au niveau des systèmes de production des éleveurs et au niveau régional.}

Table 2. Livestock system sustainability at the local and regional level.

\begin{tabular}{lccc}
\hline $\begin{array}{l}\text { Composantes } \\
\text { du développement } \\
\text { durable }\end{array}$ & $\begin{array}{c}\text { Reproductibilité } \\
\text { environnementale }\end{array}$ & $\begin{array}{c}\text { Viabilité } \\
\text { économique }\end{array}$ & $\begin{array}{c}\text { Transmission } \\
\text { intergénérationnelle }\end{array}$ \\
\hline
\end{tabular}

\section{Éléments nécessaires}

À la durabilité des systèmes de production Maintien de la des éleveurs transhumance sédentarisés

Au développement durable de la région de pâturage) animale

Cogestion des
Gestion des parcours Diversification des

(intégrité des surfaces, contrôle de la pression

Valorisation de la fumure ressources et des espaces entre groupes socioprofessionnels

Transmission entre ces groupes des savoir-faire pour la gestion des ressources naturelles
Transmission des savoirs et savoir-faire en matière d'élevage aux fils et bergers salariés (commerce du bétail développement

de la filière lait)

Maintien des effectifs bovins régionaux pour l'approvisionnement en viande, lait et bovins de trait
Sécurisation des biens et des personnes.

\author{
Renforcement \\ des relations d'échange \\ et de complémentarité \\ avec les autres acteurs \\ Amélioration de la \\ gestion des tensions \\ et conflits
}


troupeaux et donc à la possibilité de transhumer plus de 8 mois par an dans de bonnes conditions de pâturage.

\section{Des ressources naturelles à protéger}

Sur le plan régional, les ressources fourragères étant inégalement réparties dans l'espace et dans le temps, seule la transhumance permet aux éleveurs de valoriser ces ressources, afin d'assurer à leur bétail une alimentation suffisante durant toute l'année. Mais la sédentarisation familiale a modifié cette pratique. Le chef d'UP souhaite rester à proximité de sa famille et de ses champs. La scolarisation des enfants traditionnellement affectés au gardiennage impose de recourir à un ou plusieurs bergers salariés. Cela permet, entre autres, de maximiser l'accès aux divers espaces de pâturage de plus en plus morcelés en scindant le troupeau en sous-unités conduites par des bergers salariés ou par un ou plusieurs fils de la famille. Le risque majeur de cette distanciation entre la conduite du troupeau et les autres activités (agriculture, commerce, vie familiale) est la réduction des tâches de contrôle et de gestion des grands parcours (burum) pourtant essentiels au maintien de la transhumance. Cette situation peut entraîner des mises en culture d'une partie des burum et la dégradation de leur flore pastorale du fait du surpâturage.

Les éleveurs disposent d'indicateurs de dégradation des parcours par la présence de certaines herbacées comme Cassia obtusifolia, et Hyptis lanceolata. Ces indicateurs pourraient être utilisés par les bergers pour éviter les zones de parcours dégradées lors de la conduite des troupeaux afin qu'elles puissent se régénérer. Pour cela, il faut que les bergers salariés soient mieux conseillés et suivis par leurs employeurs. Dans cette région, les arbres fourragers sont de plus en plus sollicités en saison sèche pour faire face à la pénurie de biomasse fourragère et en particulier de protéines. Leur rôle est encore plus important lorsque les parcours d'herbacées sont dégradés. Les bergers sont alors amenés à distribuer au bétail des émondes d'arbres comme Terminalia laxiflora et Sterculia setigera qui auparavant n'étaient pas utilisées pour l'alimentation des bovins. Là encore, les savoirfaire doivent être transmis aux bergers dont le travail doit être contrôlé de temps à autre.
L'enjeu majeur actuel pour ces communautés est bien d'assurer le maintien de l'intégrité spatiale et de la productivité des grands parcours encore bien pourvus en ressources pastorales (les burum de Mayo Bocki, Kalgué et Dembo, etc.). Malheureusement, aucune instance de gestion réglementant l'arrivée des troupeaux transhumants n'a émergé à ce jour. La pression du bétail sur ces parcours pourrait toutefois être allégée, soit en réduisant la taille des troupeaux (ce qui semble difficilement acceptable pour des éleveurs Peuls Mbororo), soit en déclassant certaines zones de chasse (ZIC) proches de Garoua qui renferment aujourd'hui très peu de faune sauvage.

\section{Conforter l'insertion socioprofessionnelle des Mbororo}

La sédentarisation de l'habitat de ces éleveurs n'a pas suffi à conforter leur insertion dans le jeu social et politique régional. Si des progrès notables ont été obtenus dans le domaine de l'éducation de base et de la santé, ces Mbororo ont reçu peu d'appuis dans la durée pour développer leurs projets individuels (production, mise en marché) et collectifs (reconnaissance de droits sur des espaces pastoraux). De plus, le climat de violence que connaît cette région depuis 2007, et particulièrement les familles d'éleveurs, a contribué à déstabiliser ces groupes sociaux.

La nouvelle génération d'éleveurs - les fils des chefs actuels d'UP - semble bien moins intéressée par la conduite du troupeau que la génération précédente. L'élevage est considéré par les jeunes comme une activité éreintante, monotone, éloignée des lieux de vie sociale et dangereuse. Cette génération est soucieuse de diversifier ses sources de revenus (taxi moto, petit commerce, etc.) et se détache peu à peu du mode de vie pastoral. La transmission des techniques traditionnelles de conduite, d'entretien et de soin des animaux entre les générations se dégrade et se trouve menacée. Dans le cas où l'éleveur a surtout recours à des bergers salariés, ses enfants ne grandissent plus en permanence avec le bétail (le grand troupeau) et encore moins en contact étroit avec la "brousse". De ce fait, ils ont un accès moindre aux savoirs du père. Cette évolution éloignera les futurs éleveurs des lieux de transhumance et de négociation du partage de l'espace ce qui pourrait entraîner l'appauvrissement des savoirs communautaires et l'amoindrissement de leurs capacités de négociation et de gestion des ressources naturelles. Une meilleure organisation des éleveurs peut permettre de faire face à cette crise identitaire des plus jeunes. Cela implique que les Mbororo construisent des organisations professionnelles fonctionnelles afin de faciliter la transhumance et la commercialisation des produits animaux (viande, lait) et, peut-être, de développer des systèmes d'élevage intensifs moins consommateurs d'espace et complémentaires des systèmes actuels. Face à une demande d'appuis mieux structurée, les services publics et les projets de développement devront investir plus de moyens que par le passé dans des activités de formation et de conseil pour ces groupes socioprofessionnels.

\section{Conclusion}

Tout en sédentarisant leur habitat et les activités de production agricole et laitière, les éleveurs Mbororo du bassin de la Bénoué (province du Nord, Cameroun) ont dû maintenir la pratique de la transhumance pour faire face aux besoins alimentaires de leurs troupeaux. Cette sédentarisation leur a permis d'obtenir des droits fonciers sur des espaces de petite surface, de développer des liens sociaux avec d'autres acteurs ruraux et urbains, d'accéder plus facilement à certains services (école, santé, marché) et de diversifier leurs activités.

Le maintien de la transhumance est donc indispensable à la durabilité des systèmes de production des éleveurs Mbororo qui garantit l'approvisionnement en produits animaux des populations de cette région du Cameroun. Mais ces acquis pourraient être remis en question si le climat de violence qui affecte les communautés d'éleveurs Mbororo perdure. À l'avenir, cette durabilité va aussi dépendre de la capacité de ces éleveurs à s'organiser collectivement pour revendiquer des espaces suffisants de pâturage où ils assureraient une gestion effective des ressources naturelles pastorales. La question du développement durable a été ici abordée du point de vue des éleveurs, donc à l'échelle de leurs unités de production et des territoires qu'ils mobilisent. Mais ces éleveurs évoluent dans divers territoires, en fonction des saisons où ils partagent espaces 
et ressources avec d'autres producteurs. De ce fait, cette question devrait être instruite à l'échelle du bassin de la Bénoué et pour l'ensemble des systèmes de production et d'activités ruraux en vue d'aboutir à des choix de mise en valeur du territoire acceptables par tous.

\section{Références}

Boutrais J. Hautes terres d'élevage au Cameroun. Coll. Etudes et thèses. Montpellier (France) : Orstom éditions, 1996.

Benoît M. Les Bowébés du Kantoora (Sénégal). À propos de l'état pastoral. Cah Sci Hum 1988 ; $24: 379-88$.
Bonfiglioli A-M. Dudal, histoire de famille et histoire de troupeau chez un groupe de Wodaabè du Nige. Londres; Paris : Cambridge University Press; Maison des Sciences de I'Homme, 1988.

D'Aquino P. L'agropastoralisme au nord du Burkina Faso (Province du Soum) : une évolution remarquable mais encore inachevée. Autrepart 2000; $15: 29-47$.

Dugué $\mathrm{P}$, Koné $\mathrm{R}$, Koné $\mathrm{G}$, Akindes $\mathrm{F}$. Production agricole et élevage dans le centre du bassin cotonnier de Côte d'Ivoire. Cah Agric 2004 ; $13: 504-9$.

Kossoumna Liba'a N. De la mobilité à la sédentarisation: gestion des ressources naturelles et des territoires par les éleveurs Mbororo au Nord du Cameroun. Thèse de doctorat en géographie, Université Paul-Valéry Montpellier III, 2008.
Labonne M, Magrong P, Oustalet $Y$. Le secteur de I'élevage au Cameroun et dans les provinces du grand Nord. In : Jamin JY, Seiny Boukar L, Floret C, Eds. Savanes africaines : des espaces en mutation, des acteurs face à de nouveaux défis. Montpellier; N'Djaména: Cirad; Prasac, 2003 (cédérom).

Landais E. Agriculture durable : les fondements d'un nouveau contrat social ? Le Courrier de I'environnement $1998 ;(33)$ : 5-22.

Pourtier R. Les savanes africaines entre local et global: milieux, sociétés, espaces. Cah Agric $2003 ; 12: 213-8$

Seignobos C, Ousman H, Teyssier A, Weber J. Eléments d'une stratégie de développement rural pour le Grand Nord du Cameroun. Paris; Montpellier: ministères des Affaires étrangères: Cirad, 2002. 